\title{
Mt. Etna aerosol optical thickness from MIVIS images
}

\author{
Matteo Remitti $\left({ }^{1}\right)$, Sergio Pugnaghi $\left({ }^{1}\right)$ and Sergio Teggi $\left({ }^{2}\right)$ \\ (') Dipartimento di Ingegneria dei Materiali e dell'Ambiente, \\ Università degli Studi di Modena e Reggio Emilia, Modena, Italy \\ (2) Dipartimento di Ingegneria Meccanica e Civile, \\ Università degli Studi di Modena e Reggio Emilia, Modena, Italy
}

\begin{abstract}
This work focuses on the evaluation of Aerosol Optical Thickness (AOT) in Mt. Etna volcano area starting from the analysis of MIVIS VIS images. MIVIS images and ancillary data (atmospheric profiles, photometric measurements, atmospheric infrared radiances, surface temperatures, ground reflectances, $\mathrm{SO}_{2}$ abundances) were collected during the «Sicily ' $97 »$ campaign. Data elaboration was performed with extensive use of $6 \mathrm{~S}$ radiative transfer model, determining optical thickness with an inversion algorithm that uses atmospheric vertical profile, ground reflectance data and radiance measured by the first MIVIS spectrometer (channels 1-20; range 0.44-0.82 $\mu$ ). Ground reflectance is the most problematic parameter for the algorithm. In order to have a low and 'uniform' surface reflectance, only pixels located at an altitude between 2000-3000 m a.s.l. were analysed. At this altitude, AOT is very low during non-eruptive periods: at Torre del Filosofo (2920 m a.s.l.) on June 16th 1997, during one MIVIS flight, AOT at $0.55 \mu$ was 0.19 . The uncertainty about ground reflectance produces significant errors on volcanic background AOT, and in some cases the error is up to $100 \%$. The developed algorithm worked well on volcanic plume, allowing us to determine the plume related pixels' AOT. High plume AOT values minimize the problems deriving from reflectance uncertainty. Plume optical thickness shows values included in a range from 0.5 to 1.0. The plume AOT map of Mt. Etna volcano, derived from a MIVIS image of June 16th 1997, is presented.
\end{abstract}

Key words remote sensing - aerosol optical thickness - Mt. Etna volcano - MIVIS - 6S

\section{Introduction}

Mt. Etna is the largest volcano in Europe and one of the most active. It is characterised by significant lava flow and periodically strong eruptive activity. Mt. Etna volcano inject in atmosphere great quantity of gases (more than $10 \%$ of global volcanic injection for $\mathrm{H}_{2} \mathrm{O}, \mathrm{CO}_{2}$ and $\mathrm{SO}_{2}$ ) and aerosol, also during non-eruptive periods.

Mailing address: Dr. Matteo Remitti, Dipartimento di Ingegneria Materiali e Ambiente, Università degli Studi di Modena e Reggio Emilia, Via Vignolese 905, 41100 Modena, Italy; e-mail: mremitti@unimore.it
Aerosol characteristics can be estimated using remote sensing, in addition to ashes ground precipitation measurements and sampling after eruptions. Aerosol with 1-10 $\mu \mathrm{m}$ diameter can be easily identified from AVHRR or similar satellite radiometers, using TIR channels brilliance temperature difference (Prata, 1989; Wen and Rose, 1994; Ackerman, 1997; Schneider et al., 1999; Remitti et al., 2006), while liquid or solid aerosol with smaller diameter is not recognisable with such a procedure (Simpson et al., 2000).

\section{Campaign}

«Sicily '97» campaign, carried out on 10$17 / 06 / 1997$, is a part of the «Mitigation of Volcanic Risk by Remote Sensing Techniques» European project (Buongiorno et al., 1999). 


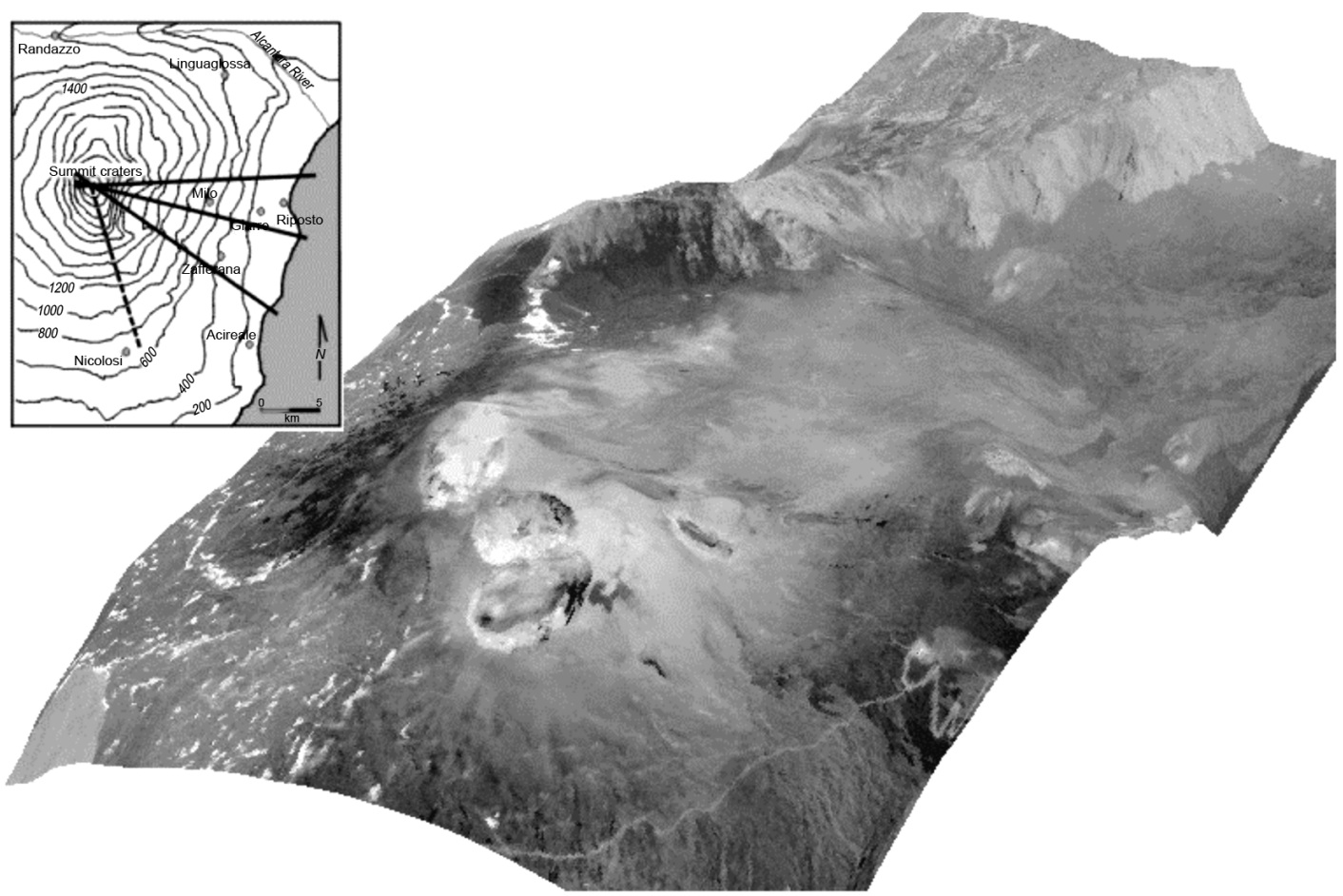

Fig. 1. Flight tracks above Mt. Etna volcano on June 16, 1997 and '3D' graphic elaboration of MIVIS RGB image.

Collected measures, integrated by data from meteorological stations, include: 1) atmospheric vertical soundings; 2) photometric measurements; 3) IR radiance measurements; 4) surface temperature measurements; 5) rocks sampling and reflectance measurements; 6) $\mathrm{SO}_{2}$ abundance measurements.

MIVIS radiometer data were obtained with repeated flights above Mt. Etna volcano. Figure 1 shows June 16 flight-tracks. The central track is aligned with plume. The dashed track is related to calibration flight.

MIVIS is a modular instrument formed by four spectrometers (Daedalus, 1996), with 102 channels ranging from VIS to TIR. Visible channels (1-20) range from 0.44 to $0.82 \mu$. Viewing angle during sampling is $1.6436 \mathrm{mrad}$, there are 755 pixels per line, and scan angle is $35.55^{\circ}$.
Figure 1 shows a '3D' graphic elaboration of MIVIS RGB image obtained using channels 14, 7 and 4. Elevation is taken from Digital Elevation Model (Buongiorno et al., 1999). The source image is $2 \mathrm{D}$, therefore in this elaboration the plume results inevitably 'squashed' on the ground.

\section{Simulations}

$6 \mathrm{~S}$ is a radiative transfer model written in Fortran language (Vermote et al., 1997). The list of inputs includes: 1) viewing geometry (date, angles, altitude, ...); 2) atmospheric profile (measured, standard, hybrid, ...); 3) aerosol type and concentration (refraction index, size distribution, AOT, ...); 4) spectral conditions (monochromatic, range, response function, ...); 5) soil 
characterization (homogeneous or inhomogeneous, reflectance, brdf, ...).

Outputs include: 1) input values; 2) integrated values (apparent reflectance, simulated radiance to sensor, total gas transmittance); 3) components (irradiance at ground (direct, scattered, environmental), radiance to sensor (atmospheric intrinsic radiance, environmental radiance, target radiance), apparent reflectance to sensor, response function integral, ...); 4) trasmittances (gas, Rayleigh, aerosol, spherical albedo, optical thickness, phase function, single scattering albedo).

After verification of the relationship between simulated radiance and geometrical inputs (target and sensor altitudes, angles) and reflectance, many simulations were run, modifying aerosol parameters (abundance, type, size; Watson and Oppenheimer, 2000) and studying the effects on radiance to sensor, trying to build an inversion algorithm to determine AOT from MIVIS image.

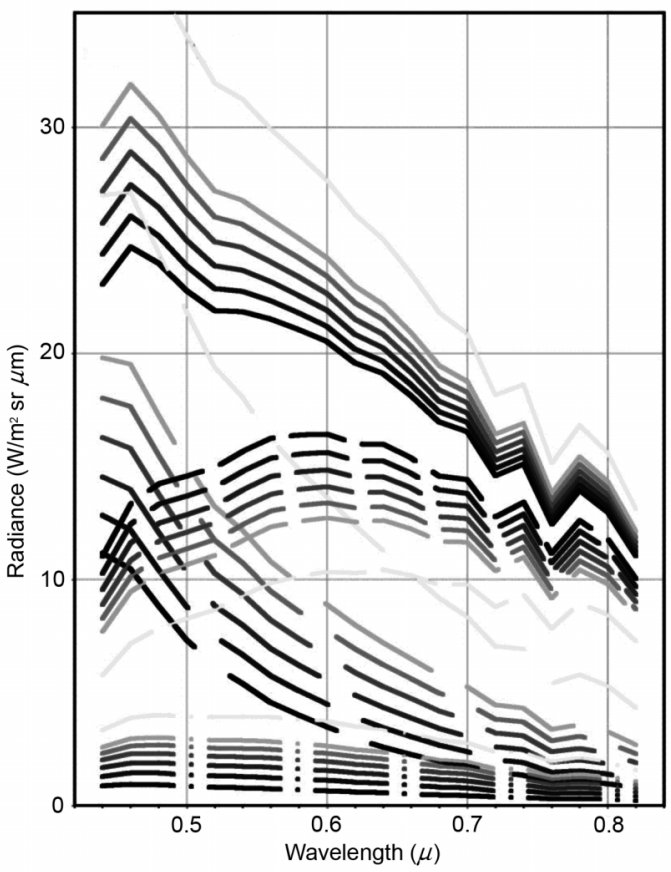

Fig. 2. $6 \mathrm{~S}$ simulated radiances for a lava area: total simulated radiance (solid line), atmospheric intrinsic radiance (long dashed line), background radiance (short dashed line) and target radiance (dash-dot line).
Radiance to sensor tends to grow in correspondence of higher AOT, even if atmospheric transmittance decreases. Because of the very low ground reflectance (lava, volcanic ashes), atmospheric reflectance contribution to total radiance to sensor is higher than target plus environment reflected radiance. In fact, atmospheric intrinsic radiance grows if $\tau$ increases, and overcomes the decrease of soil reflected radiance.

Figure 2 shows total simulated radiance (solid line) and its three components («atmospheric intrinsic radiance» (long dashed line), «background radiance» (short dashed line) and «target radiance» (dash-dot line); Vermote et al., 1997) for a Mt. Etna lava area and different AOT values. Target and environment contributions decrease if $\tau$ grows, while atmospheric reflected radiance increases and makes total radiance do the same.

For a snow area, because of higher reflectance, ground reflected radiance grows more than atmospheric intrinsic radiance decrease, therefore total simulated radiance is higher for lower AOT.

Apart from direct or inverse relationships, the difference between lava and snow areas follows from the incidence of atmospheric contribution. For lava or ashes, the atmospheric reflectance contribution is higher than others, and separates distinctly lines for different AOTs (in the next example, up to $50 \%$ between first and last simulations on channel 5), while for snow opposite contributions minimize the gap, and lines result very close or even indistinguishable. In addition, there is a higher variation in measured radiance from snow areas, and this makes it impossible to discriminate AOT from MIVIS radiance.

In figure 3 thick solid lines include MIVIS radiance range of variation $(L \pm \sigma)$ for a snow area (2750 $\mathrm{m}$ a.s.1., 100 pixels). The ten dashed lines are outputs for $6 \mathrm{~S}$ simulations using different $\tau$, starting from very low values to typical industrial area values, unattainable for this altitude in Mt. Etna area.

High snow reflectance and high variability in region of interest makes it impossible to determine AOT, because every line (even with extremely thin or thick AOT) is inside the standard deviation of measured radiance. 


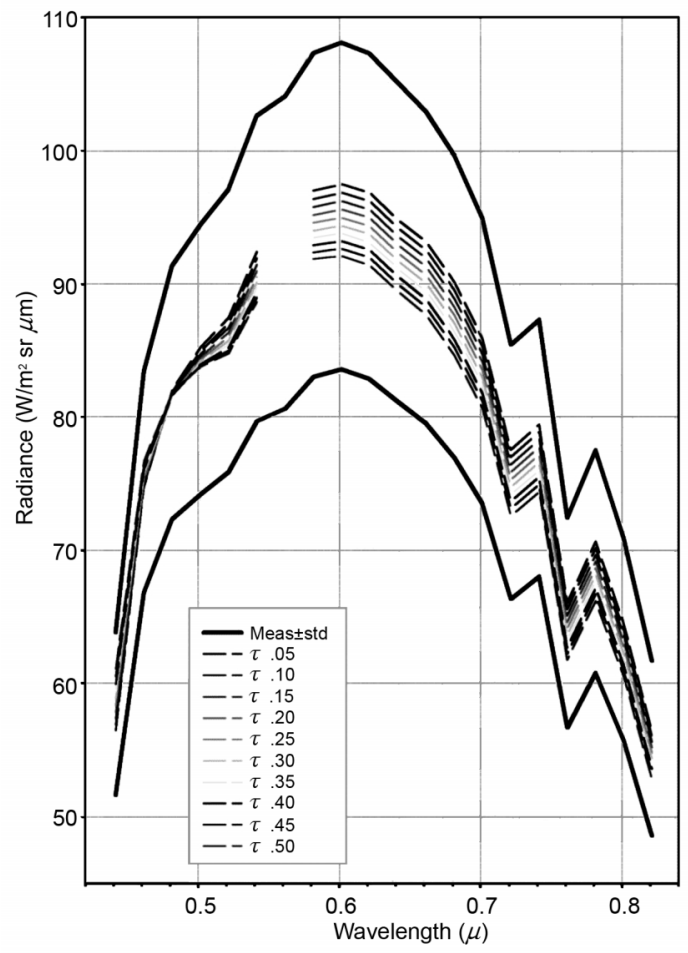

Fig. 3. MIVIS measured radiance range of variation $(L \pm \sigma)$ for a snow area (thick solid lines) and $6 \mathrm{~S}$ simulated radiances using different AOT (dashed lines).

For lava areas, instead, AOT determination from MIVIS radiance is possible: in fig. 4 the two thick solid lines $(L \pm \sigma)$ univocally identify $\tau=0.2$, with clear line identification especially for lower wavelengths (where the gap between lines is wider, while standard deviation is approximately constant on 20 channels).

Low ground reflectance is an unconditional requirement for AOT determination starting from measured and simulated radiance.

So, starting from: 1) MIVIS spectral radiance image; 2) geometrical characteristics (view and sun angles, altitude); 3) atmospheric vertical profile; 4) ground reflectance measurements (as seen before, correct aerosol estimation needs low ground reflectance); and 5) approximate aerosol size distribution, a correct estimate of aerosol optical thickness can be ob-

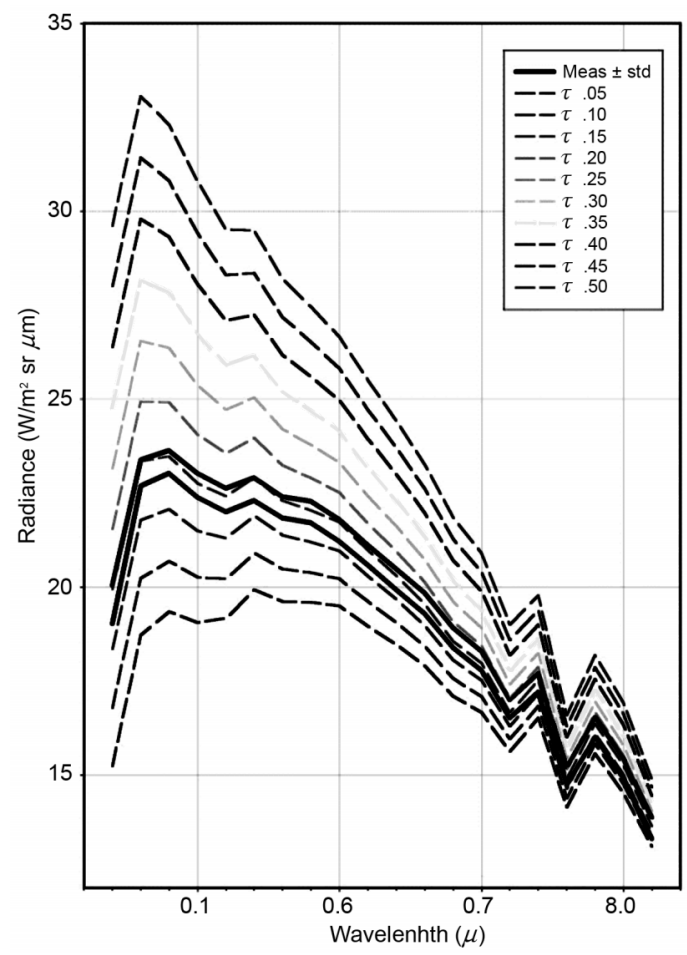

Fig. 4. MIVIS measured radiance range of variation $(L \pm \sigma)$ for a lava area (thick solid lines) and 6S simulated radiances using different AOT (dashed lines).

tained, using $6 \mathrm{~S}$ radiative model and procedure explained above (Remitti, 2001).

\section{Reflectance}

Reflectance is the most problematic parameter: its uncertainty inhibits aerosol determination outside plume. Good results are instead obtained in the plume area, where the optical thickness is higher.

In order to have a low and mostly uniform surface reflectance, only pixels located at an altitude between 2000-3000 m a.s.l. were analysed.

Constant reflectance in single channel is used in all simulations, obtaining a short computational time and no negative effect on results' quality (channels are narrow and selected 
response function is square). In multichannel simulation $\tau$ is value in full width at half maximum of every single channel.

Spectral reflectance of rock samples (FieldSpec laboratory data) from the «Sicily '97» campaign was analysed. Lines were grouped in 'families', starting from information about type of sample, but differences between lines were significant inside each 'family', too. Some observations: 1) lines in second half of range are nearly horizontal; 2) reflectance does not decrease along with the increase of wavelength; 3 ) in some lines there is a slope change at $700 \mathrm{~nm}$ (due to vegetation); 4) ashes have a lower reflectance than lava, contrary to human observation, in which lava appears darker; 5) reflectance is always very low $(\tau<0.1)$.

Spectral reflectance of small areas in the Etna image is obtained using 6S in «atmospheric correction» mode (Vermote et al., 1997), which, starting from radiance, gives as output soil reflectances. Some observations: 1) lines have similar shape; 2) in UVA range reflectance values are very low (in some cases there is an error with negative values); 3) ashes have higher reflectance than lava.

Measured reflectance (FieldSpec) is very different from the MIVIS/6S-obtained.

Ashes and lavas are rocks of the same type (basalt). Ash fields, observed from a distance, seem to be lighter than lava, but it is surprisingly difficult to distinguish a lava rock in an ash field. An explanation of this can be found in 'scale effect', due to differences in observation distances.

For ashes, MIVIS views an ash field as a homogeneous surface, smooth and regular, therefore characterised by a higher reflectance. FieldSpec, instead, views from a few dozen centimetres, appreciating irregularity in structure (shadows, sloped faces, ...) that determines a reduction of apparent reflectance.

For lavas, instead, MIVIS views the complex structure of flows (fractures, holes, shadows) that, after 6S elaboration, gives a low reflectance; while in laboratory FieldSpec views uniform and compact surfaces (in some case rocks are cut), and tends to overestimate reflectance.

Significant evidence of this is given from MIVIS measurements of jeep path. This path, consisting of trodden ashes, has no difference in material from the surrounding terrain, but in the MIVIS image, particularly in some channels, is clearly identifiable.

Figure 5a shows some reflectances (measured and simulated): lava spectral reflectances are plotted in black, and ashes reflectance in grey. FieldSpec data are plotted with dotted lines (lava) and dash-dot lines (ashes); MIVIS data are plotted with solid lines (lava) and dashed lines (ashes).

Some observations: 1) for ashes, FieldSpec gives very low reflectance, with a small spectral variation, while MIVIS/6S gives higher values; 2) for lava, FieldSpec reflectances are highest in the graph, while MIVIS/6S values are very low, with an opposite effect compared with ashes; 3) lines shape for FieldSpec and MIVIS/6S is clearly different.

In these conditions it is very difficult to hypothesize a ground reflectance measurement providing results that can be used from flying sensor (Remitti, 2001). Possibly, unconventional measurements (holes for lava, different sizes for ashes) and/or measurements from a longer distance could be useful. Otherwise, it is possible to study transformation from FieldSpec and MIVIS/6S reflectance.

\section{Aerosol map}

6S simulation on plume pixels, starting from six areas characterised by different levels of apparent transparency, produces a significant result: the shape of each line does not show significant differences from the others. The only effect is a higher radiance, due to a higher atmospheric reflectance. This is a very important observation: it is correct to infer that radiation passes through plume in both directions, producing a scheme that can be correctly modelled with $6 \mathrm{~S}$, and excluding mirror-like light reflection from plume (which is not compatible with $6 \mathrm{~S}$ radiative scheme).

Aerosol map elaboration is realised with a procedure organized according to the following schedule. 1) 6S simulations are realised to evaluate model sensitivity and select steps, obtaining $5^{\circ}$ for zenith angle (from $0^{\circ}$ to $35^{\circ}$ per side, 15 


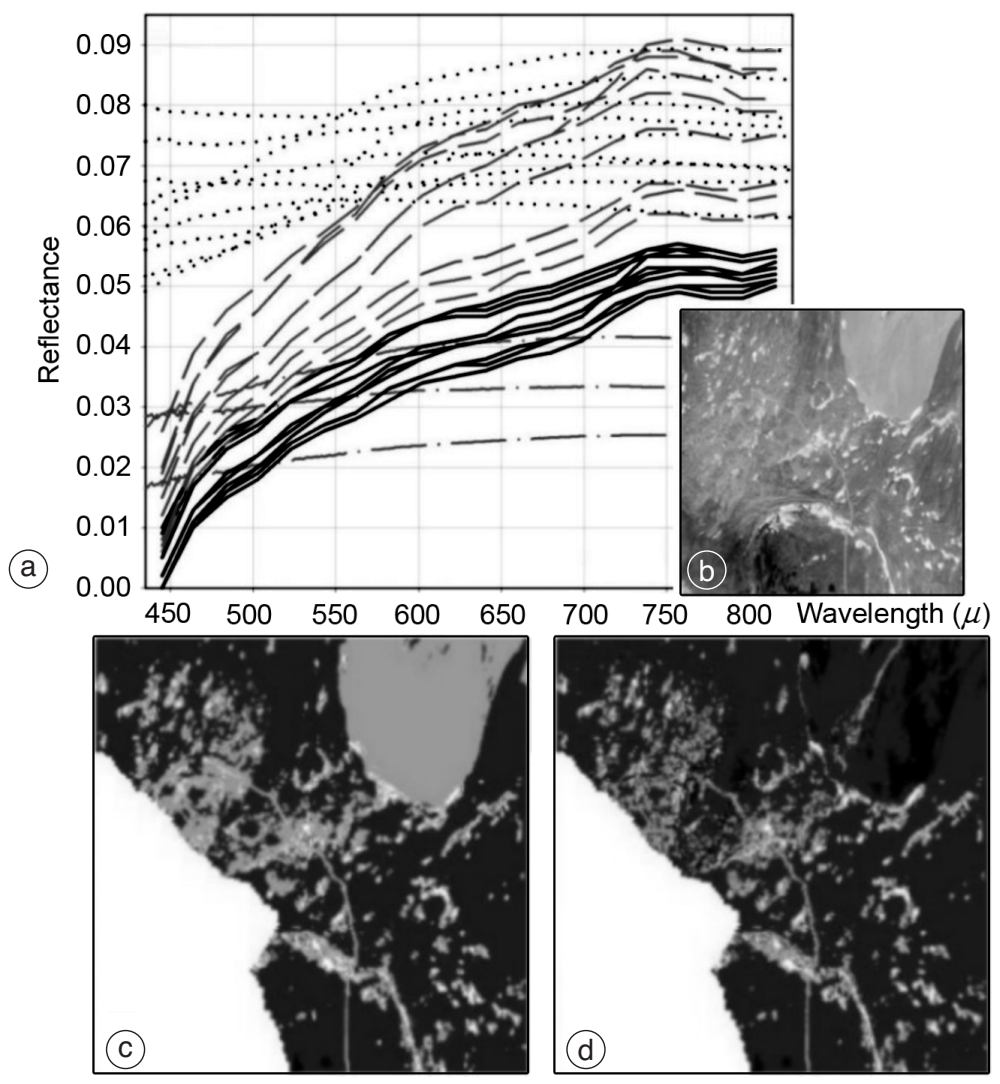

Fig. 5a-d. a) Measured and simulated reflectances: MIVIS/6S lava (solid lines), FieldSpec lava (dotted lines), MIVIS/6S ashes (dashed lines), FieldSpec ashes (dash-dot lines); b) part of MIVIS RGB image; c) related AOT map using one spectral reflectance; d) related AOT map using two-values reflectance mask.

steps), $100 \mathrm{~m}$ for altitude (from 2000 to $3000 \mathrm{~m}$ a.s.1., 11 levels), and different values for aerosol optical thickness (always $\tau 0=0$ and $\tau n=n * \Delta \tau$, with $n=1, \ldots, 10,11$ steps). 2) Reflectances are obtained from $6 \mathrm{~S}$ in «atmospheric correction mode», calculating mean values from the same type of areas and applying little correction. As shown, FieldSpec reflectance is not usable. 3) $6 \mathrm{~S}$ loops are organized and processed: for each reflectance, simulation for all configurations (20 channels, 16 angles, 11 quotes, 11 aerosol optical thickness values) are made. 4) From each output file a table with input data and correspondent output simulated radiance for MIVIS channels is compiled.
A Fortran-written program analyses every pixel in the image, and 1) evaluates coordinates and chooses corresponding angles; 2) extracts altitude from DEM file and approximates it to simulation altitude level; 3) selects the portion of table reproducing input data; 4) extracts from MIVIS image radiance values; 5) chooses aerosol optical thickness values that minimize standard deviation between simulated and measured radiances

$$
D_{t}^{2}=\sum_{i=1}^{20}\left(L_{m, i}-L_{s, i}\right)^{2}
$$

6) builds an aerosol map, in which an AOT ob- 
tained from procedure above is associated with every pixel (Remitti, 2001).

Outside plume the procedure (using $\tau$ from 0 to 0.5 ) gives correct values, but has high relative errors. Uncertainty about soil reflectance inhibits good determination of aerosol, and aerosol map reproduces every local characteristic of the input MIVIS image (ash fields, different lava apparent colour, jeep path, ...), giving out different AOT for pixels with same atmospheric conditions. In the plume area problems due to surface reflectance are reduced because of the higher atmospheric reflection, but the high aerosol content 'saturates' output in AOT.

A new elaboration is realised, using $\tau$ from 0 to 2.5 . In this case it is possible to discrimi- nate different AOT inside plume, and the other pixels give a uniform aerosol value. Some pixels, corresponding to snow, again show 'saturation': these areas have high radiance, and $6 \mathrm{~S}$, starting from same input soil reflectance and interpreting radiance as an effect of atmospheric reflectance, produces a high AOT value.

The first tests are realised on an area outside the plume, which includes every typical surface characteristics: different types of lava, ash, snow, jeep path. Figure 5b,c shows starting MIVIS image and results from elaboration using one spectral reflectance. Ash field has different AOT value from lava: this is obviously incorrect (areas are contiguous and at the same altitude) and due exclusively to different soil reflectance. To avoid

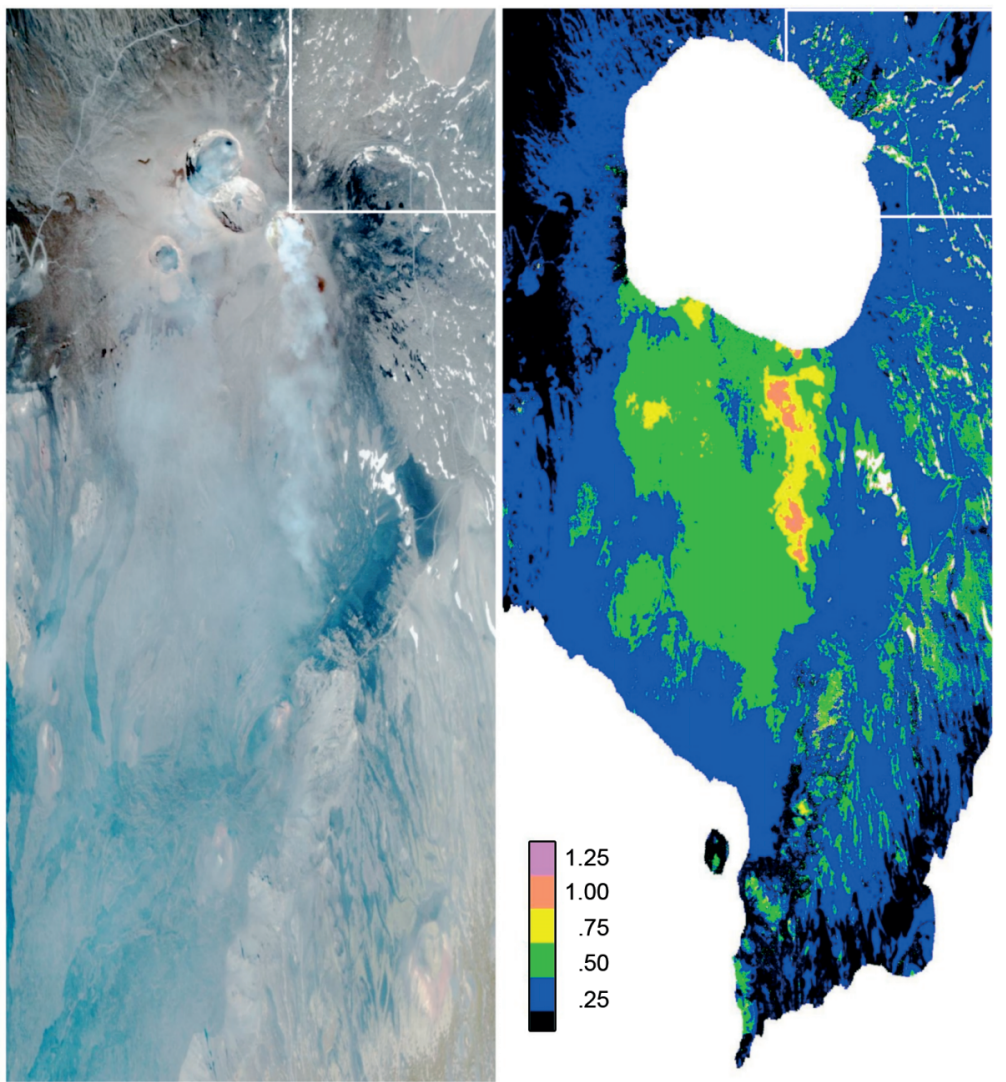

Fig. 6. AOT map (blue $\tau=0.25$, green $\tau=0.50$, yellow $\tau=0.75$, orange $\tau=1.00$, pink $\tau=1.25$ ) and MIVIS RGB image. 
this problem, the procedure is modified to have a «reflectance mask» as input. For each pixel the algorithm selects correct reflectance, choosing the related $6 \mathrm{~S}$ output table for checking simulated and measured radiances. Figure $5 d$ shows the AOT map of the same area, obtained using twovalues reflectance mask: only pixels related to jeep path or snow (that 'saturate') have higher values of aerosol optical thickness.

In this approach, the chosen criteria to create aerosol maps are very important. Some tests show that building a reflectance map using only two reflectance values (input reflectance are obtained from $6 \mathrm{~S}$ in «atmospheric correction mode») yields good ground modelling for exposed purposes. Many tests have been run, combining the thresholds on two different channels (or on two different channel ratios) or using a threshold on two channels (or two different channel ratios) linear combination. Selected reflectance mask was obtained after an application of b4 mask on linear combination of ratio of MIVIS channel 2 to channel 5 and of channel 2 to channel 19. This mask allows discrimination of all test areas.

Results of the inversion algorithm are shown in fig. 6, together with the MIVIS RGB image (Remitti, 2001). Plume has AOT values between 0.5 and 1 (green (0.50), yellow (0.75), orange (1.00) areas), while pixels outside plume show uniform $\tau=0.25$, which is in good accordance with the value at Torre del Filosofo $(\tau=0.19$ at $2920 \mathrm{~m}$ from photometric measurements).

The reflectance map correctly describes soil characteristics, and only for a few pixels are AOT values different because of reflectance. Snow areas produce 'saturation', but they are easily identifiable.

\section{Conclusions}

The developed inversion algorithm worked well on Mt. Etna volcanic plume, determining the plume related pixels' AOT over ground (while satellite techniques for AOT evaluation are tested typically over sea; Moula et al., 2002; Ignatov and Stowe, 2002a,b). High plume AOT values (together with altitude and reflectance 'uniformity') minimize the problems deriving from reflectance uncertainty, which entails high relative error outside plume. Plume optical thickness in MIVIS image of June 16th, 1997 shows values included in a range from 0.5 to 1.0. Good tests of this inversion procedure could have some significant forthcoming application, using satellite images.

Hyperion radiometer on EO-1 satellite (USGS-NASA, in orbit from 2000; Pearlman et al., 2001) has on board a MIVIS-like radiometer (220 bands from 0.4 to $2.5 \mu$ ), and it supplies images that can be processed with a similar algorithm allowing AOT evaluation and characterization.

The results can be useful for analysing the time evolution of volcanic emission too: volcanic gases tend to condensate around condensation nuclei (e.g., from $\mathrm{SO}_{2}$ to sulphuric acid, Porter et al., 2002), and AOT data from inversion algorithm can help determining condensing time and conditions. All these data are important in vulcanology.

\section{REFERENCES}

ACKerman, S.A. (1997): Remote sensing aerosols using satellite infrared observations, J. Geophys. Res., 102 (14), 17069-17079.

Buongiorno, M.F., L. Merucci, F. Doumaz, S. Salvi, M. P. Bogliolo, S. Pugnaghi, S. Teggi, S. Corradini, L. Lombroso, A. Sterni, T. CALTABiano and V. CARrÉre (1999): MVRRS campaign: MIVIS mission on Sicilian volcanoes and ground measurements, Quad. Geofis., 7, pp. 90.

DaedAlus (1996): MIVIS Technical Specifications, Daedalus Enterprises.

Ignatov, A. and L. Stowe (2002a): Aerosol retrievals from individual AVHRR channels, Part I. Retrieval algorithm and transition from Dave to $6 \mathrm{~S}$ radiative transfer model, J. Atmos. Sci., 59 (3), 313-334.

IgnAtov, A. and L. Stowe (2002b): Aerosol retrievals from individual AVHRR channels, Part II. Quality control, probability distribution functions, information content, and consistency checks of retrievals, J. Atmos. Sci., 59 (3), 335-362.

Moula, M., J. Verdebout and H. Eva (2002): Aerosol optical thickness retrieval over the Atlantic Ocean using GOES imager data, Phys. Chem. Earth, 27 (35), 15251531.

Pearlman, J., C. Segal, P. Clancy, N. Nelson, P. Jarecke, M. OnO, D. Beiso, L. LiaO, K. YoKoyama, S. CARMAN, B. Browne, L. ONG and S. Ungar (2001): The EO-1 Hyperion Imaging Spectrometer (available on line at: http://eo1.usgs.gov/documents/hyperionpub.asp).

Porter, J.N., K.A. Horton, P.J. Mouginis-Mark, B. Lienert, S. K. Sharma, E. LaU, A.J. Sutton, T. Elias 
and C. OPPENHEIMER (2002): Sun photometer and LIDAR measurements of the plume from the Hawaii Kilauea Volcano Pu'u O'o vent: aerosol flux and $\mathrm{SO}_{2}$ lifetime, Geophys. Res. Lett., 29 (16), 1783.

PratA, A.J. (1989): Infrared radiative transfer calculation for volcanic ash clouds, Geophys. Res. Lett., 16 (11), 1293-1296.

RemitTi, M. (2001): Sviluppo di tecniche di telerilevamento per il monitoraggio di aerosol e nubi vulcaniche, $\mathrm{Te}$ si di Laurea (Università degli Studi di Modena e Reggio Emilia).

Remitti, M., S. Pugnaghi, S. Teggi and F. Parmiggiani (2006): Retrieval of tropospheric ash clouds of Mt. Etna from AVHRR data, in Proceedings of the Mid Term Meeting of the GNV Project on Remote Sensing, 24-25 January 2002, Catania, Quad. Geofis. (in press).

SchneIDER, D.J., W.I. Rose, L.R. CoKE, G.J.S. Bluth, I.E. SPROD and A.J. KRUEGER (1999): Early evolution of a stratospheric volcanic eruption cloud as observed with TOMS and AVHRR, Geophys. Res. Lett., 104 (4), 40374050.

Simpson, J.J., G. Hufford, D. Pieri and J. Berg (2000): Failures in detecting volcanic ash from a satellite-based technique, Remote Sensing Environ., 72, 192-217.

Vermote, E., D. Tanré, J.L. Deuzé, M. Herman and J.J. MorCRETTE (1997): Second Simulation of the Satellite Signal in the Solar Spectrum (6S), 6S User Guide Version 2 (University of Maryland, Dept. Of Geograph/ Laboratoire d'Optique Atmospherique, Lille).

WATSON, I.M. and C. OpPEnHeIMER (2000): Particle size distributions of Mount Etna's aerosol plume constrained by sun photometry, J. Geophys. Res., 105 (8), 9823-9829.

WEN, S. and W.I. Rose (1994): Retrieval of sizes and total masses of particles in volcanic clouds using AVHRR bands 4 and 5, J. Geophys. Res., 99 (3), 5421-5431. 